\title{
Blood Purification of Bilirubin and Bile Acids by Plasma Exchange for Patients with Liver Failure
}

\author{
Shuji Matsubara, Kenji Okabe, Kiyoaki Ouchi and \\ Yasuo Owada \\ The First Department of Surgery, Tohoku University School \\ of Medicine, Sendai 980
}

\begin{abstract}
Matsubara, S., Okabe, K., Ouchi, K. and Owada, Y. Blood Purification of Bilirubin and Bile Acids by Plasma Exchange for Patients with Liver Failure. Tohoku J. exp. Med., 1986, 150 (1), 83-90 — To evaluate the efficacy of blood purification by plasma exchange in patients with liver failure, blood levels of total bilirubin and total bile acids, known to be a part of protein-bound toxins, were measured before and after plasma exchange. With one plasma volume exchange the percent reduction was 39 for bilirubin and only 25 for bile acids. The declining of the reduction efficiency appeared to be well correlated with the prolongation of the treatment time for bilirubin and the augmentation of the initial concentration for bile acids. These data suggested that the equilibration between intra-and extra-vascular pool takes place slowly for bilirubin and comparatively rapidly for bile acids, though they are both mostly bound to albumin in the blood. In addition, the presence of protein-bound toxins like bile acids, predominantely accumulated in the tissue, may make it more difficult to support patients with liver failure by only blood purificaiton therapy with plasma exchange. - plasma exchange; protein-bound toxins; bilirubin; bile acids; liver failure
\end{abstract}

In order to remove augmented noxious substances, various treatment modalities with extracorporeal circulation have been employed for the treatment of liver failure. Most work has been concentrated on hemoperfusion and techniques such as dialysis, hemofiltration or plasma exchange. Although all the putative toxins in liver failure have not been totally clarified yet, evidence suggests they mostly exist in the blood as macromolecules bound to plasma proteins (Zieve 1975; Kuster 1976). For that reason, all procedures except plasma exchange do not seem to meet the primary purpose of eliminating all the toxins. Plasma exchange can be a feasible measure for hepatic support, because it not only removes small molecular substances but also reduces whole plasma proteins. However, there has been some scattering in the reported results of clinical plasma exchange, since it has been carried out empirically without scientific basis. An objective evaluation of plasma exchange is necessary to

Received May 13, 1986; accepted for publication July 14, 1986. 
optimize the treatment regimen for liver failure. The present study was undertaken to assess the efficacy and limitation of plasma exchange in reducing the protein-bound toxins such as bilirubin and bile acids accumulating in the blood in case of liver failure.

\section{Materials and Methods}

The study included 9 patients with liver diseases who underwent plasma exchange. The diagnosis of the patients were postoperative liver failure $(n=4)$, liver failure caused by the exacerbation of liver cirrhosis $(n=2)$, secondary biliary cirrhosis $(n=2)$, and intrahepatic cholestasis $(n=1)$. Plasma exchange was carried out using a membrane plasma separator (Centry TPE system; Cobe Laboratories, Inc., Lakewood, Co, USA). The exchanged plasma volume was between $2.5 \mathrm{~L}$ to $6.2 \mathrm{~L}$. As the substitution fluid, fresh frozen plasma was predominantly used for liver failure patients to supply coagulation factors, and in other cases, plasma protein fraction (Plasmanate; Cutter Japan, Kobe) was principally employed.

In order to evaluate the reduction efficacy of plasma solutes by plasma exchange, blood samples were taken prior to the treatment and immediately at termination. Pre and post change was measured 57 times for total bilirubin in all patients, and 20 times for total bile acids in three patients whose plasma was exchanged only with the plasma protein fraction (Table 1). Total bilirubin was assayed by an autoanalyzer using the alkaliazobilirubin method and total bile acids were measured with fluorimetric and enzymatic assay.

The reduction ratio $(R)$ of bilirubin and bile acids was calculated by the following equation:

$R=C / C o$, where $C o$ is the initial concentration and $\mathrm{C}$ is the final concentration. The range of reduction ratio, (1-R), was normalized with the exchanged plasma volume ratio and expressed as the normalized percent reduction $(\mathrm{NZ} \% \mathrm{RD})$, which indicates the percentage of the reduction range per one plasma volume exchange, using the following equation:

$\mathrm{NZ} \% \mathrm{RD}=\frac{1-R}{V / V o} \times 100$, where $V o$ is the circulating plasma volume and $V$ is the exchanged plasma volume. Vo was determined empirically with a nomogram using the body weight, the height, and the hematocrit (Buffaloe et al. 1983). In addition, the

TABle 1. Patient population of liver diseases treated by plasma exchange

\begin{tabular}{lccllcc}
\hline \multirow{2}{*}{ Case } & Age & Sex & & \multicolumn{2}{c}{ Disease } & Number of data point \\
\cline { 4 - 6 } & & & & Bilirubin & Bile acids \\
\hline Z.M. & 63 & M & PLF* & 12 & - \\
K.A. & 55 & M & PLF with LC† & 5 & - \\
H.S. & 54 & M & PLF with LC & 2 & - \\
Y.K. & 60 & M & PLF & 8 & - \\
K.K. & 53 & M & LFł with LC & 3 & - \\
Y.A. & 53 & M & LF with LC & 2 & - \\
M.T. & 28 & M & Secondary biliary cirrhosis & 10 & 8 \\
Y.S. & 50 & M & Secondary biliary cirrhosis & 10 & 8 \\
K.I. & 71 & F & Intrahepatic cholestasis & 5 & 4 \\
& Total & & & 57 & 20 \\
\hline
\end{tabular}

*post operative liver failure; †liver cirrhosis; łliver failure. 
theoretical reduction ratio $\left(R^{\prime}\right)$ according to the single compartment theory, which assumes the intravascular mass is completely isolated from the remainder of the body, was calculated by the following equation:

$$
R^{\prime}=\exp (-V / V o)
$$

The difference between $R$ and $R^{\prime}$ was expressed as the percent error from the predicted value $(\% \mathrm{ERR})$ according to the following equation :

$$
\% \mathrm{ERR}=\left(1-R^{\prime} / R\right) \times 100
$$

In order to assess the factors having influence upon the negative increment of the range of $\% \mathrm{ERR}$, the initial concentration of each plasma solute and the length of treatment time were compared with each $\%$ ERR value. All data were expressed as the mean \pm s.D. Data analysis was performed using the paired, two-tailed Student's $t$-test.

\section{RESULTS}

The pre and post values of plasma exchange were $25.5 \pm 14.5 \mathrm{mg} / 100 \mathrm{ml}$ and $11.9 \pm 6.6 \mathrm{mg} / 100 \mathrm{ml}$ for total bilirubin (the average was $R=0.47$ ), and $99.8 \pm 48.2$ $\mathrm{nmole} / \mathrm{ml}$ and $75.9 \pm 34.7 \mathrm{nmole} / \mathrm{ml}$ for total bile acids (the average was $R=0.76$ ). The reduction range of bilirubin and bile acids with the exchange of one plasma volume are summarized and illustrated in Fig. 1 as NZ\%RD. The reduction was by $39.1 \pm 5.9 \%$ for total bilirubin and by $25.1 \pm 8.8 \%$ for total bile acids. The reduction range of bile acids was significantly smaller than that of bilirubin $(p<$ $0.05)$. In addition, the average $\% \mathrm{ERR}$ was $-84.6 \pm 46.8 \%$ for bilirubin and $-135 \pm 54.9 \%$ for bile acids. The differences in the reduction rate between the theoretical value and the actual value were larger in bile acids than in bilirubin with a statistical significance of $p<0.05$. As shown in Fig. 2, the $\%$ ERR of total

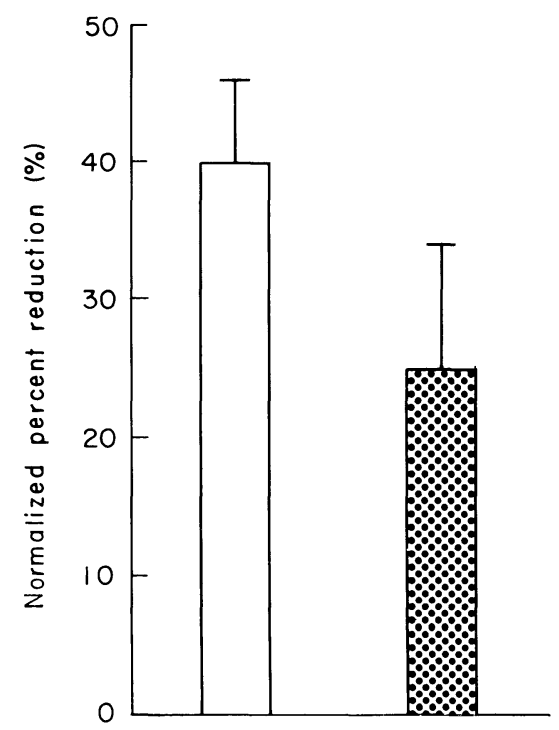

Fig. 1. Normalized percent reduction of total bilirubin $(\square, n=55)$ and total bile acids $(\because \therefore, n=20)$ by plasma exchange. 


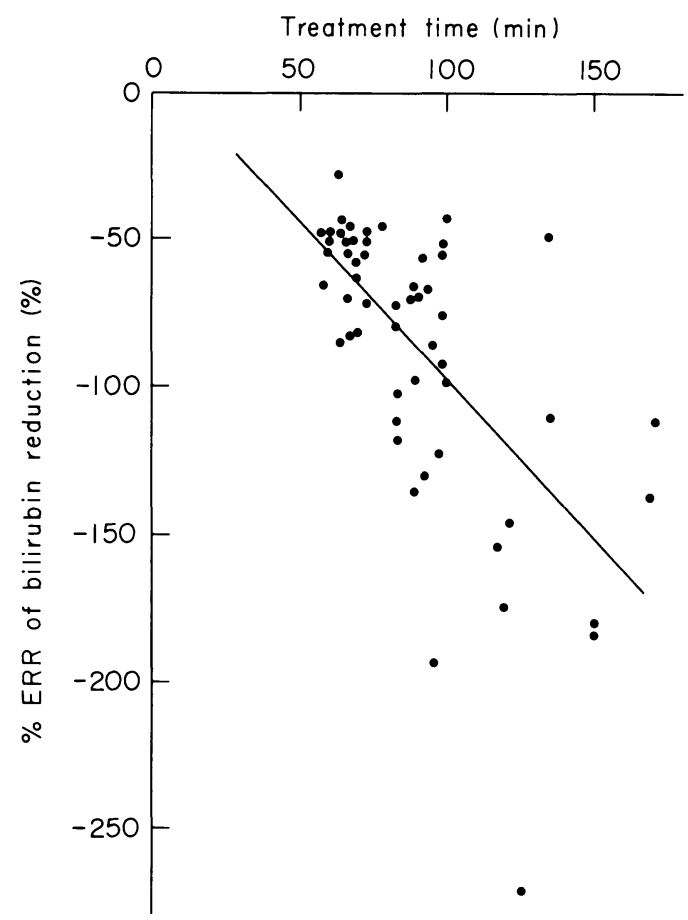

Fig. 2. Correlation between percent error of total bilirubin reduction and treatment time. $r=-0.622(p<0.01)$.

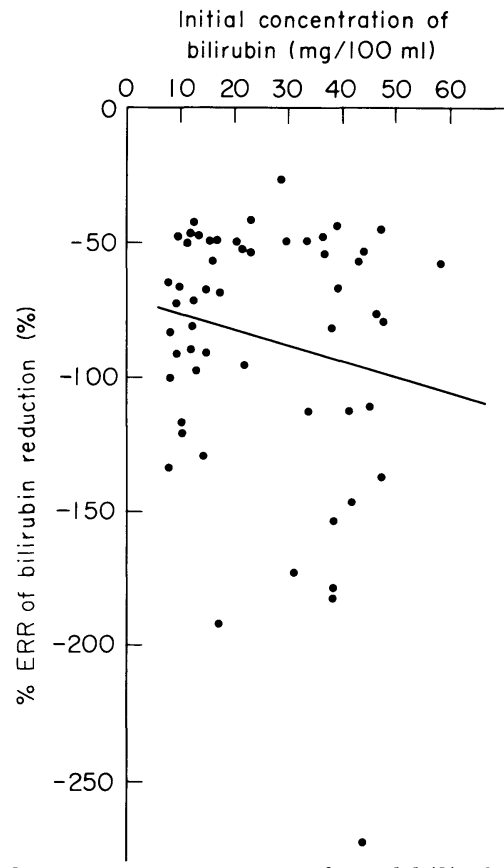

Fig. 3. Correlation between percent error of total bilirubin reduction and initial concentration. $r=-0.172$. 


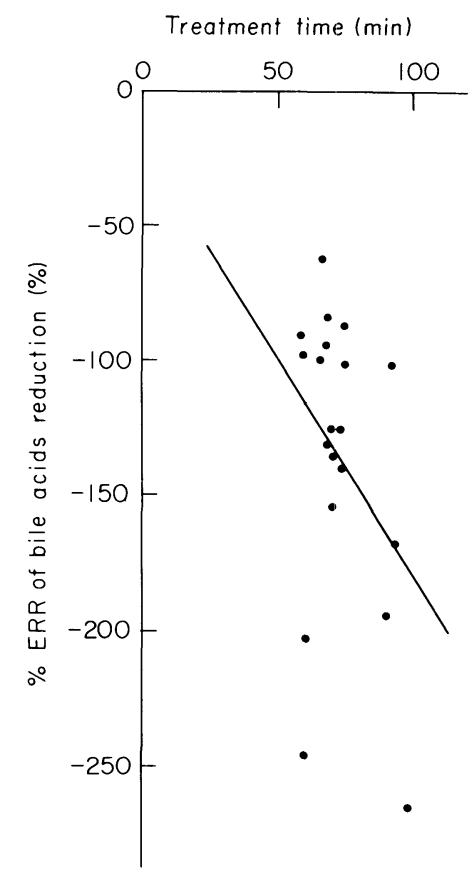

Fig. 4. Correlation between percent error of total bile acids reduction and treatment time. $r=-0.343$.

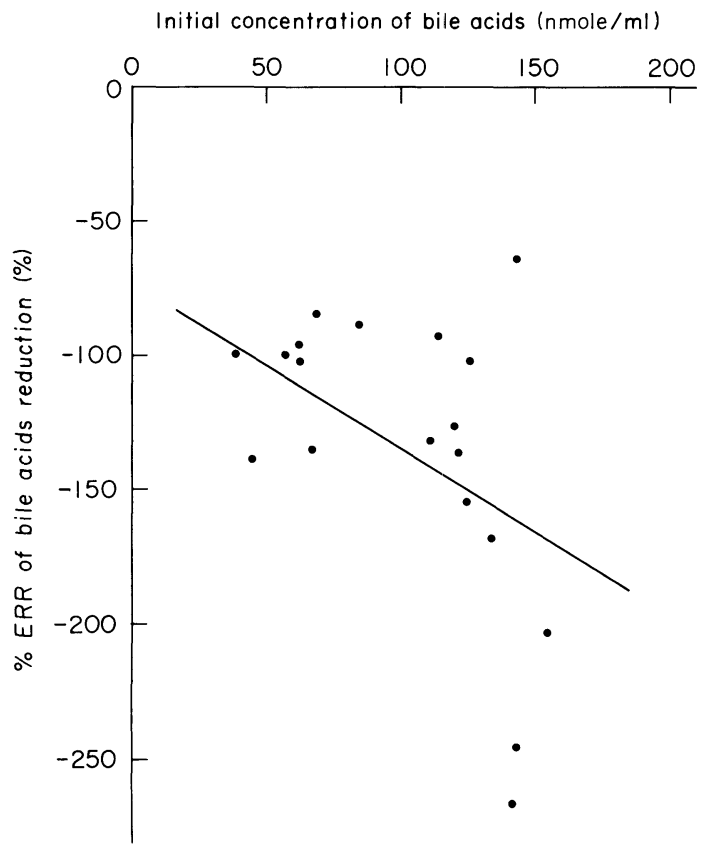

Fig. 5. Correlation between percent error of total bile acids reduction and initial concentration. $r=-0.475(p<0.05)$. 
bilirubin was negatively correlated with the treatment time with a correlation coefficient of $r=-0.622$. The efficacy of bilirubin purification declined with the prolongation of the treatment time. On the other hand, Fig. 3 illustrates that the $\%$ ERR of bilirubin was poorly correlated with the initial concentration as shown by a correlation coefficient of $r=-0.172$. The error of the reduction ratio in bilirubin was found to be independent of the concentration before the treatment. As illustrated in Fig. 4, the \%ERR of total bile acids showed a low correlatation with the treatment time, the correlation coefficient being $r=-0.343$. The error of the reduction ratio in bile acids was not found to be dependent on the length of the treatment. However, as shown in Fig. 5, there was a significant negative correlation between the $\% \mathrm{ERR}$ of bile acids and their initial concentration, with a correlation coefficient of $r=-0.475(p<0.05)$, that is, the reduction efficiency of bile acids declined according to the increase of the initial concentrations.

\section{Discussion}

Using techniques of extracorporeal circulation, a variety of blood purification systems have been applied to the patients with liver failure in the past. Kiley et al. (1956) were the first to employ the dializer for the removal of blood ammonia. Chang (1972) and Gazzard et al. (1974) carried out hemoperfusion over coated charcoal in patients with hepatic coma. Opolon et al. (1976) performed hemodialysis with a high-permeable membrane with polyacrylonitorile to remove middle molecule toxins, and plasma exchange was first used by Lepore and Martel (1967). The efforts with hemoperfusion and hemodialysis resulted in an overall survival rate of no more than some twenty percent, which does not significantly exceed the results obtained by conservative treatment.

Although the augmenting putative toxins in liver failure have not been totally identified yet, according to the current medical knowledge and technologies, plasma exchange may be the most feasible procedure for purifying the blood. Because of the indiscriminate removal of whole plasma components, plasma exchange can reduce all noxious metabolites, especially macromolecular protein-bound toxins which are unable to be removed by other techniques. However, even with plasma exchange the overall survival remains around thirty percent. There may be two reasons for such unsatisfactory results. First of all, the regeneration of necrotic liver mass may not actually take place as much as is expected, and secondary the exchanged plasma volume may not be sufficient enough to support the damaged liver. The amount of plasma to be removed has been apparently determined by individual experience or personal conviction without a scientific basis (Derksen et al. 1984). Therefore, it is necessarry to assess correctly the efficacy of plasma exchange in liver failure in order to optimize the treatment regimen.

The present study evaluated the reduction efficiency of two protein-bound toxins, bilirubin and bile acids, which accumulate when there is liver 
insufficiency. In addition, the causative factors producing a decline in the reducing efficacy were investigated. Though both bilirubin and bile acids are bound to albumin in the blood, there was a significant discrepancy observed in the purification efficiency. The range of percent reduction by one plasma volume exchange $(\mathrm{NZ} \% \mathrm{RD})$ was 40 for total bilirubin and only 25 for total bile acids. These data suggest that more than 2 or 3 times greater volumes of plasma should be exchanged to reduce protein-bound toxins like bile acids sufficiently.

The error between the actual reduction and the theoretical value, calculated according to the single compartment theory, was examined and expressed by $\%$ ERR. The negative increase of $\% \mathrm{ERR}$ indicates that there is a considerable mass transfer of the plasma solute occurring from the tissue pool into the intravascular space. The average $\%$ ERR was shown to be $-85 \%$ for total bilirubin and $-135 \%$ for total bile acids. The large negative error of total bile acids illustrates that the blood levels of bile acids may be regulated more by the equilibration from the extravascular pool. In addition, \% ERR was negatively correlated with the length of the treatment time of bilirubin and the initial concentration of bile acids. Bilirubin may move from the extravascular pool into the blood relatively slowly, so that $\%$ ERR may increase negatively in accordance with the prolongation of the treatment time. On the other hand, the equilibration of bile acids between the intra and extravascular spaces may occur rapidly and the size of the extravascular pool may be much larger than the intravascular pool. Therefore, it is conceivable that the high initial concentration, mostly equal to the tissue concentration, produces the negative increase of $\% \mathrm{ERR}$. In the present study the change of bile acids was investigated only in the patients with cholastasis to exclude the influence of bile acids contained in fresh frozen plasma. Because the augmentation of bile acids in blood is known to be much striking, this tendency may be more magnified in the case of liver failure.

These results indicate that the equilibration between blood and tissue does not occur in the same manner with bilirubin and bile acids despite the fact that both are bound to albumin in the blood. Presumably, the survival rate of the patients with liver insufficiency treated with plasma exchange can not be improved due to the presence of protein-bound toxins like bile acids, which are predominantly accumulated in the extravascular space. More aggressive and intensive plasma exchange may be necessary to increase it.

\section{References}

1) Buffaloe, G.W., Dau, P.C., Erickson, M.A., Fosburg, M.T., Grossman, L. \& Taft, E.G. (1983) Technical considerations. In: Therapeutic Plasma Exchange, Disease Compendium edited by P.C. Dau, COBE Laboratories, Inc., Lakewood, pp. 3-21.

2) Chang, T.M.S. (1972) Hemoperfusion over microencapsulated absorbent in a patient with hepatic coma. Lancet, 2, 1371-1372.

3) Derksen, R.H.W., Schurman, H.J., Gmelig May Ling, F.H.J., Struyvenberg, A. \& Kater, L. (1984) The efficacy of plasma exchange in the removal of plasma compo- 
nents. J. Lab. clin. Med., 104, 346-354.

4) Gazzard, B.G., Portmann, B., Weston, M.J., Langlen, P.G., Murray-Lyon, I.M., Dunlop, E.H., Flax, H., Mellon, P.J., Record, C.O., Ward M.B. \& Williams, R. (1974) Charcoal hemoperfusion in the treatment of fulminant hepatic failure. Lancet, $\mathbf{1}$, 1301-1307.

5) Kiley, J.E., Welch, H.F., Pender, J.C. \& Welch, C.S. (1956) Removal of blood ammonia by hemodialysis. Proc. Soc. exp. Biol. Med., 91, 489-490.

6) Kuster, G.G. R. (1976) Introduction. In : Hepatic Support in Acute Liver Failure, edited by G.G.R. Kuster, Charles C. Thomas Publisher, Springfield, pp. 3-12.

7) Lepore, M.J. \& Martel, A.J. (1967) Plasmapheresis in hepatic coma. Lancet, 2, 771772 .

8) Opolon, P., Rapin, J-R., Huguet, C., Granger, A., Delorme, M-L., Boschat, M. \& Sausse, A. (1976) Hepatic failure coma treated by polyacrylonitrile membrane hemodialysis. Trans. Amer. Soc. Artif. Intern. Organs, 22, 701-710.

9) Zieve, L. (1975) Metabolic abnormalities in hepatic coma and potential toxins to be removed. In : Artificial Liver Support, edited by R. Williams \& I.M. Murray-Lyon, Pitmal Medical Co., Great Britain, pp. 11-26. 